\title{
Divergent Cesàro Means of Jacobi-Sobolev Expansions
}

\author{
Bujar Xh. Fejzullahu \\ Faculty of Mathematics and Sciences \\ University of Prishtina \\ Nëna Terezë 5 \\ 10000 Prishtinë - Kosovo \\ bujarfej@uni-pr.edu
}

Received: February 2, 2007

Accepted: November 22, 2007

\section{ABSTRACT}

Let $\mu$ be the Jacobi measure supported on the interval $[-1,1]$. Let introduce the Sobolev-type inner product

$$
\langle f, g\rangle=\int_{-1}^{1} f(x) g(x) d \mu(x)+M f(1) g(1)+N f^{\prime}(1) g^{\prime}(1),
$$

where $M, N \geq 0$. In this paper we prove that, for certain indices $\delta$, there are functions whose Cesàro means of order $\delta$ in the Fourier expansion in terms of the orthonormal polynomials associated with the above Sobolev inner product are divergent almost everywhere on $[-1,1]$.

Key words: Jacobi-Sobolev type polynomials, Fourier expansion, Cesàro mean.

2000 Mathematics Subject Classification: 42C05, $42 \mathrm{C} 10$.

\section{Introduction}

Let $d \mu(x)=(1-x)^{\alpha}(1+x)^{\beta} d x, \alpha>-1, \beta>-1$, be the Jacobi measure supported on the interval $[-1,1]$. Let $f$ and $g$ functions in $L^{2}(\mu)$ such that there exists the first derivative in 1 . We can introduce the discrete Sobolev-type inner product

$$
\langle f, g\rangle=\int_{-1}^{1} f(x) g(x) d \mu(x)+M f(1) g(1)+N f^{\prime}(1) g^{\prime}(1)
$$


where $M \geq 0, N \geq 0$. We denote by $\left\{q_{n}^{(\alpha, \beta)}\right\}_{n \geq 0}$ the sequence of orthonormal polynomials with respect to the inner product (1) (see [1]). These polynomials are known in the literature as Jacobi-Sobolev type polynomials. For $M=N=0$, the classical Jacobi orthonormal polynomials appear. We will denote them $\left\{p_{n}^{(\alpha, \beta)}\right\}_{n \geq 0}$.

For every function $f$ such that $\left\langle f, q_{n}^{(\alpha, \beta)}\right\rangle$ exists for $n=0,1, \ldots$, the Fourier expansion in Jacobi-Sobolev type polynomials is

$$
\sum_{n=0}^{\infty} c_{n}(f) q_{n}^{(\alpha, \beta)}(x)
$$

where

$$
c_{n}(f)=\left\langle f, q_{n}^{(\alpha, \beta)}\right\rangle .
$$

The Cesàro means of order $\delta$ of the Fourier expansion (2) are defined by (see $[9$, p. $76-77])$

$$
\sigma_{N}^{\delta} f(x)=\sum_{n=0}^{N} \frac{A_{N-n}^{\delta}}{A_{N}^{\delta}} c_{n}(f) q_{n}^{(\alpha, \beta)}(x)
$$

where $A_{k}^{\delta}=\left(\begin{array}{c}k+\delta \\ k\end{array}\right)$.

In this contribution we will prove that there are functions such that their Cesàro means of order $\delta$ diverge almost everywhere on $[-1,1]$. A similar result, when $M=N=0$, has been obtained in [6].

Notice that, for an appropriate function $f$, the study of the convergence of Fourier series in terms of the polynomials associated to the Sobolev inner product

$$
\langle f, g\rangle=\int_{-1}^{1} f(x) g(x) d \mu(x)+M f(c) g(c)+N f^{\prime}(c) g^{\prime}(c)
$$

when $c \in[-1,1]$ has been presented $[7]$ and when $c \in(1, \infty)$ in $([3,4])$ some analog results have been deduced.

Throughout this paper positive constants are denoted by $c, c_{1}, \ldots$ and they may vary at every occurrence. The notation $u_{n} \sim v_{n}$ means $c_{1} \leq u_{n} / v_{n} \leq c_{2}$ for sufficiently large $n$, and by $u_{n} \cong v_{n}$ we mean that the sequence $u_{n} / v_{n}$ converges to 1 .

\section{Jacobi-Sobolev type polynomials}

Some basic properties of the polynomials $q_{n}^{(\alpha, \beta)}$ (see [1]) that we will need in the sequel, are given in below:

$$
q_{n}^{(\alpha, \beta)}(x)=A_{n} p_{n}^{(\alpha, \beta)}(x)+B_{n}(x-1) p_{n-1}^{(\alpha+2, \beta)}(x)+C_{n}(x-1)^{2} p_{n-2}^{(\alpha+4, \beta)}(x)
$$

where 
(i) if $M>0$ and $N>0$ then

$$
A_{n} \cong-c n^{-2 \alpha-2}, \quad B_{n} \cong c n^{-2 \alpha-2}, \quad C_{n} \cong 1,
$$

(ii) if $M=0$ and $N>0$ then

$$
A_{n} \cong \frac{-1}{\alpha+2}, \quad B_{n} \cong 1, \quad C_{n} \cong \frac{1}{\alpha+2},
$$

(iii) if $M>0$ and $N=0$ then

$$
\begin{gathered}
A_{n} \cong c n^{-2 \alpha-2}, \quad B_{n} \cong 1, \quad C_{n} \cong 0 . \\
\left|q_{n}^{(\alpha, \beta)}(1)\right| \sim \begin{cases}n^{-\alpha-3 / 2} & \text { if } M>0, N \geq 0, \\
n^{\alpha+1 / 2} & \text { if } M=0, N \geq 0 .\end{cases} \\
\left(q_{n}^{(\alpha, \beta)}\right)^{\prime}(1) \sim n^{-\alpha-7 / 2} \text { if } M \geq 0, N>0 . \\
\max _{x \in[-1,1]}\left|q_{n}^{(\alpha, \beta)}(x)\right| \sim n^{\beta+1 / 2} \quad \text { if }-1 / 2 \leq \alpha \leq \beta .
\end{gathered}
$$

for $\alpha \geq-1 / 2, \beta \geq-1 / 2$, and $n \geq 1$.

The asymptotic behavior of $q_{n}^{(\alpha, \beta)}$, when $x \in[-1+\epsilon, 1-\epsilon]$ and $\epsilon>0$, is given by

$$
q_{n}^{(\alpha, \beta)}(x)=s_{n}^{\alpha, \beta}(1-x)^{-\alpha / 2-1 / 4}(1+x)^{-\beta / 2-1 / 4} \cos (k \theta+\gamma)+O\left(n^{-1}\right),
$$

where $x=\cos \theta, k=n+\frac{\alpha+\beta+1}{2}, \gamma=-(\alpha+1) \frac{\pi}{2}$, and $\lim _{n \rightarrow \infty} s_{n}^{\alpha, \beta}=\left(\frac{2}{\pi}\right)^{1 / 2}$.

The Mehler-Heine formula for Jacobi orthonormal polynomials is (see [8, Theorem 8.1.1 and (4.3.4)]

$$
\lim _{n \rightarrow \infty}(-1)^{n} n^{-\beta-1 / 2} p_{n}^{(\alpha, \beta)}\left(\cos \left(\pi-\frac{z}{n}\right)\right)=2^{-\frac{\alpha+\beta}{2}}(z / 2)^{-\beta} J_{\beta}(z),
$$

where $\alpha, \beta$ are real numbers and $J_{\beta}(z)$ is the Bessel function. This formula holds uniformly for $|z| \leq R$, for $R$ a given positive real number.

From (9)

$$
\lim _{n \rightarrow \infty}(-1)^{n} n^{-\beta-1 / 2} p_{n}^{(\alpha, \beta)}\left(\cos \left(\pi-\frac{z}{n+j}\right)\right)=2^{-\frac{\alpha+\beta}{2}}(z / 2)^{-\beta} J_{\beta}(z)
$$

holds uniformly for $|z| \leq R, R$ a fixed positive real number, and uniformly on $j \in N \cup\{0\}$. 
Lemma 1.1. Let $\alpha, \beta>-1$ and $M, N \geq 0$. There exists a positive constant $c$ such that

$$
\lim _{n \rightarrow \infty}(-1)^{n} n^{-\beta-1 / 2} q_{n}^{(\alpha, \beta)}\left(\cos \left(\pi-\frac{z}{n}\right)\right)=c(z / 2)^{-\beta} J_{\beta}(z),
$$

uniformly for $|z| \leq R, R>0$ fixed.

Proof. Here we will only analyze the case when $M=0$ and $N>0$. The proof of the other cases can be done in a similar way. From (3) we have

$$
\begin{gathered}
(-1)^{n} n^{-\beta-1 / 2} q_{n}^{(\alpha, \beta)}\left(\cos \left(\pi-\frac{z}{n+j}\right)\right)=A_{n}(-1)^{n} n^{-\beta-1 / 2} p_{n}^{(\alpha, \beta)}\left(\cos \left(\pi-\frac{z}{n+j}\right)\right) \\
-B_{n}\left(\cos \left(\pi-\frac{z}{n+j}\right)-1\right)(-1)^{n-1} n^{-\beta-1 / 2} p_{n-1}^{(\alpha+2, \beta)}\left(\cos \left(\pi-\frac{z}{n+j}\right)\right) \\
+C_{n}\left(\cos \left(\pi-\frac{z}{n+j}\right)-1\right)^{2}(-1)^{n-2} n^{-\beta-1 / 2} p_{n-2}^{(\alpha+4, \beta)}\left(\cos \left(\pi-\frac{z}{n+j}\right)\right)
\end{gathered}
$$

where $j \in N \cup\{0\}$.

Finally, if $n \rightarrow \infty$ and using (3) and (10) we get

$$
\begin{aligned}
\lim _{n \rightarrow \infty}(-1)^{n} & n^{-\beta-1 / 2} q_{n}^{(\alpha, \beta)}\left(\cos \left(\pi-\frac{z}{n+j}\right)\right) \\
& =\left(-\frac{1}{\alpha+2} 2^{-\frac{\alpha+\beta}{2}}+2 \cdot 2^{-\frac{\alpha+\beta+2}{2}}+\frac{1}{\alpha+2} 4 \cdot 2^{-\frac{\alpha+\beta+4}{2}}\right)\left(\frac{z}{2}\right)^{-\beta} J_{\beta}(z) \\
& =2^{-\frac{\alpha+\beta}{2}}\left(\frac{z}{2}\right)^{-\beta} J_{\beta}(z) .
\end{aligned}
$$

For every function $f$ such that $\left\langle f, q_{n}^{(\alpha, \beta)}\right\rangle$ exists for $n=0,1, \ldots$, the Fourier-Sobolev coefficients of the series (2) can be written as

$$
c_{n}(f)=\left\langle f, q_{n}^{(\alpha, \beta)}\right\rangle=c_{n}^{\prime}(f)+M f(1) q_{n}^{(\alpha, \beta)}(1)+N f^{\prime}(1)\left(q_{n}^{(\alpha, \beta)}\right)^{\prime}(1),
$$

where

$$
c_{n}^{\prime}(f)=\int_{-1}^{1} f(x) q_{n}^{(\alpha, \beta)}(x)(1-x)^{\alpha}(1+x)^{\beta} d x .
$$

Next, we will estimate the following integral involving Jacobi-Sobolev type polynomials

$$
\int_{-1}^{1}\left|q_{n}^{(\alpha, \beta)}(x)\right|^{q}(1-x)^{\alpha}(1+x)^{\beta} d x
$$

where $1 \leq q<\infty$. For $M=N=0$ the calculation of this integral appears in [8, p. 391, Exercise 91] (see also [5, (2.2)]).

First we compute an upper bound for this integral: 
Theorem 1.2. Let $M \geq 0$ and $N \geq 0$. For $\alpha \geq-1 / 2$

$$
\int_{0}^{1}(1-x)^{\alpha}\left|q_{n}^{(\alpha, \beta)}(x)\right|^{q} d x= \begin{cases}O(1) & \text { if } 2 \alpha>q \alpha-2+q / 2 \\ O(\log n) & \text { if } 2 \alpha=q \alpha-2+q / 2 \\ O\left(n^{q \alpha+q / 2-2 \alpha-2}\right) & \text { if } 2 \alpha<q \alpha-2+q / 2\end{cases}
$$

For $\beta \geq-1 / 2$

$$
\int_{-1}^{0}(1+x)^{\beta}\left|q_{n}^{(\alpha, \beta)}(x)\right|^{q} d x= \begin{cases}O(1) & \text { if } 2 \beta>q \beta-2+q / 2 \\ O(\log n) & \text { if } 2 \beta=q \beta-2+q / 2 \\ O\left(n^{q \beta+q / 2-2 \beta-2}\right) & \text { if } 2 \beta<q \beta-2+q / 2\end{cases}
$$

Proof. From (7), for $q \alpha+q / 2-2 \alpha-2 \neq 0$, we have

$$
\begin{aligned}
\int_{0}^{1}(1-x)^{\alpha}\left|q_{n}^{(\alpha, \beta)}(x)\right|^{q} d x= & O(1) \int_{0}^{\pi / 2} \theta^{2 \alpha+1}\left|q_{n}^{(\alpha, \beta)}(\cos \theta)\right|^{q} d \theta \\
= & O(1) \int_{0}^{n^{-1}} \theta^{2 \alpha+1} n^{q \alpha+q / 2} d \theta \\
& +O(1) \int_{n^{-1}}^{\pi / 2} \theta^{2 \alpha+1} \theta^{-q \alpha-q / 2} d \theta \\
= & O\left(n^{q \alpha+q / 2-2 \alpha-2}\right)+O(1),
\end{aligned}
$$

and for $q \alpha+q / 2-2 \alpha-2=0$ we have

$$
\int_{0}^{1}(1-x)^{\alpha}\left|q_{n}^{(\alpha, \beta)}(x)\right|^{q} d x=O(\log n) .
$$

For the proof of the second part we can proceed in a similar way.

Now, a technique similar to the used in [8, Theorem 7.34] yields:

Theorem 1.3. Let $M \geq 0$ and $N \geq 0$. For $\beta>-1 / 2$

$$
\int_{-1}^{0}(1+x)^{\beta}\left|q_{n}^{(\alpha, \beta)}(x)\right|^{q} d x \sim n^{q \beta+q / 2-2 \beta-2}
$$

where $\frac{4(\beta+1)}{2 \beta+1}<q<\infty$.

Proof. For the proof of this theorem it is enough to find a lower bound for the integral. 
Let $\beta \geq-1 / 2, M \geq 0$ and $N \geq 0$. According to Lemma 1.1, we have

$$
\begin{aligned}
\int_{\pi / 2}^{\pi}(\pi-\theta)^{2 \beta+1}\left|q_{n}^{(\alpha, \beta)}(\cos \theta)\right|^{q} d \theta & >\int_{\pi-1 / n}^{\pi}(\pi-\theta)^{2 \beta+1}\left|q_{n}^{(\alpha, \beta)}(\cos \theta)\right|^{q} d \theta \\
& =\int_{0}^{1}(z / n)^{2 \beta+1} \mid q_{n}^{(\alpha, \beta)}\left(\left.\cos (\pi-z / n)\right|^{q} n^{-1} d z\right. \\
& \cong c \int_{0}^{1}(z / n)^{2 \beta+1} n^{q \beta+q / 2}\left|(z / 2)^{-\beta} J_{\beta}(z)\right|^{q} n^{-1} d z \\
& \sim n^{q \beta+q / 2-2 \beta-2} .
\end{aligned}
$$

\section{Divergent Cesàro means of Jacobi-Sobolev expansions}

If the expansion (2) is Cesàro summable of order $\delta$ on a set, say $E$, of positive measure in $[-1,1]$, then from $[9$, Theorem 3.1.22] (see also [6, Lemma 1.1]) we get

$$
\left|c_{n}(f) q_{n}^{(\alpha, \beta)}(x)\right|=O\left(n^{\delta}\right), \quad x \in E .
$$

From the Egorov's theorem there exists a subset $E_{1} \subset E$ of positive measure such that

$$
\left|c_{n}(f) q_{n}^{(\alpha, \beta)}(x)\right|=O\left(n^{\delta}\right)
$$

uniformly for $x \in E_{1}$. Hence, from (8), we have

$$
\left|n^{-\delta} c_{n}(f)\left(\cos (k \theta+\gamma)+O\left(n^{-1}\right)\right)\right| \leq c
$$

uniformly for $x=\cos \theta \in E_{1}$. Using the Cantor-Lebesgue Theorem, (see [6, subsection 1.5] as well as $[9$, p. 316]), we get

$$
\left|\frac{c_{n}(f)}{n^{\delta}}\right| \leq c, \quad \forall n \geq 1 .
$$

Now we will prove our main result:

Theorem 2.1. Let $\alpha, \beta, p$, and $\delta$ be given numbers such that

$$
\begin{aligned}
\beta & >-1 / 2, & -\frac{1}{2} & \leq \alpha \leq \beta, \\
1 \leq p & <\frac{4(\beta+1)}{2 \beta+3}, & 0 & \leq \delta<\frac{2 \beta+2}{p}-\frac{2 \beta+3}{2} .
\end{aligned}
$$

There exists $f \in L^{p}(\mu)$, supported on $[-1,0]$, whose Cesàro means $\sigma_{N}^{\delta} f(x)$ are divergent almost everywhere on $[-1,1]$. 
Proof. Assume that

$$
1 \leq p<\frac{4(\beta+1)}{2 \beta+3}, \quad \delta<\frac{2 \beta+2}{p}-\frac{2 \beta+3}{2}
$$

For $q$ conjugate to $p$, from the last inequalities, we get

$$
\frac{4(\beta+1)}{2 \beta+1}<q \leq \infty, \quad \delta<\beta+\frac{1}{2}-\frac{2 \beta}{q}-\frac{2}{q} .
$$

For the linear functional $c_{n}^{\prime}(f)=\int_{-1}^{1} f(x) q_{n}^{(\alpha, \beta)}(x) d \mu(x)$, from the uniform boundedness principle, (6) and Theorem 1.3, it follows that there is $f \in L^{p}(\mu)$, supported on $[-1,0]$, such that

$$
\frac{c_{n}^{\prime}(f)}{n^{\delta}} \rightarrow \infty, \quad \text { when } \quad n \rightarrow \infty .
$$

Hence, from (4), (5), and (11), we obtain

$$
\frac{c_{n}(f)}{n^{\delta}} \rightarrow \infty, \quad \text { when } \quad n \rightarrow \infty .
$$

Since this result is contrary with $(12) \sigma_{N}^{\delta} f(x)$ is divergent almost everywhere.

Remark 2.2. Using formulae in [2], which relate the Riesz and Cesàro means of order $\delta \geq 0$, we conclude that Theorem 2.1 also holds for Riesz means.

\section{References}

[1] M. Alfaro, F. Marcellán, and M. L. Rezola, Estimates for Jacobi-Sobolev type orthogonal polynomials, Appl. Anal. 67 (1997), no. 1-2, 157-174.

[2] J. J. Gergen, Summability of double Fourier series, Duke Math. J. 3 (1937), no. 2, 133-148.

[3] F. Marcellán, B. P. Osilenker, and I. A. Rocha, On Fourier series of Jacobi-Sobolev orthogonal polynomials, J. Inequal. Appl. 7 (2002), no. 5, 673-699.

[4] On Fourier-series of a discrete Jacobi-Sobolev inner product, J. Approx. Theory 117 (2002), no. 1, 1-22.

[5] C. Markett, Cohen type inequalities for Jacobi, Laguerre and Hermite expansions, SIAM J. Math. Anal. 14 (1983), no. 4, 819-833.

[6] C. Meaney, Divergent Cesàro and Riesz means of Jacobi and Laguerre expansions, Proc. Amer. Math. Soc. 131 (2003), no. 10, 3123-3128.

[7] I. A. Rocha, F. Marcellán, and L. Salto, Relative asymptotics and Fourier series of orthogonal polynomials with a discrete Sobolev inner product, J. Approx. Theory 121 (2003), no. 2, 336-356.

[8] G. Szegö, Orthogonal polynomials, Colloquium Publications of the American Mathematical Society, vol. 23, American Mathematical Society, Providence, R.I., 1975.

[9] A. Zygmund, Trigonometric series: Vols. I, II, Cambridge University Press, London, 1968. 